\title{
MODELING AND SIMULATION STANDARDS PRODUCTS AND PROCESSES
}

\author{
William F. Flanigan, Jr. \\ Center for Standards \\ Defense Information Systems Agency \\ Reston, VA 22090-5006, U.S.A.
}

\begin{abstract}
This paper provides an overview of some modeling and simulation (M\&S) standards products and processes (SP\&PS) recently developed or emerging at the Department of Defense (DoD) level. Recent SP\&Ps include the formation of a Standards Infrastructure Team to address broad-based standards and standardization needs, guidelines for establishing an infrastructure strategy to support the development of synthetic environments, and an electronic, information-system component containing mid-level summaries of $M \& S$ protocols/standards for DoD acquisition program managers (APMs). Emerging SP\&Ps include a dial-in, expert system for developing M\&S standards profiles by DoD APMs, an electronic M\&S standards framework, a "fast track" for the adoption of standards by DoD, seamless interoperability between $M \& S$ information systems, and an M\&S Data Administration effort for data accessibility and reuse.
\end{abstract}

\section{INTRODUCTION}

Defense-related modeling and simulation $(M \& S)$ tools have been around for decades. However, a series of recent events (e.g., Defense initiatives, Defense Science and Technology Thrusts (S\&TT), budget downsizing, and Defense conversion reinvestment and transition assistance) are coalescing to elevate $M \& S$ derivatives (such as advanced distributed simulations and synthetic environments) to keystone status in Defense acquisitions, combined-forces training and readiness, production and logistics, research, development, test and evaluation, and analysis. In parallel with this process has been an explosion in information technology.

To exploit information technology in support of the Defense $M \& S$ community, a family of standardsrelated products and processes have been developed or are emerging to promote and achieve system interoperability and broad information dissemination within the M\&S community. Some of these are briefly described below.

\section{RECENTLY DEVELOPED PRODUCTS AND PROCESSES}

A Standards Infrastructure Team (SIT) has been established through the auspices of the Defense Modeling and Simulation Office (DMSO). It is one of ten Infrastructure Task Force teams originally formed by DMSO to determine shortfalls and opportunities that cut across the entire M\&S community. SIT's principal role is to provide the focal point for information and guidance in standards and standards-related matters for the $M \& S$ community (since standards impact all $M \& S$ infrastructure areas, SIT could be viewed as the "infra" in M\&S infrastructure). Current activities include: identifying and documenting unaddressed, broad-based standards and standardization needs and requirements of current/emerging $M \& S$ developers/users within the $M \& S$ community; initiating and/or evaluating candidate DMSO infrastructure proposals on the basis of satisfying critical shortfalls; providing and supporting the broadest dissemination of information to the $M \& S$ community on standards and standards-related matters; stimulating the migration of M\&S standards to lower-cost, vendorneutral, open-system environments and interconnections; promoting the cultural change needed to optimize the use of advanced distributed simulations and inter-operable synthetic environments so that cost reductions can be achieved; and fostering Defense conversion and dual use for National Security and the National Economy. SIT is establishing an electronic bulletin board on the DMSO Information System (IS). It will provide a number of services for the M\&S community including (1) a continually-updated source for information and guidance on standards, protocols, and standardization, (2) a means to query the collective expertise of SIT members (through an interactive questions-and-answers process), and (3) an M\&S standards forum. SIT membership is expanding and ultimately will consist of individuals who represent DoD, Federal, private-sector, and academic M\&S developers, implementers, and users. This means that SIT and its "customers" will become one and the same. You are invited to apply for membership by contacting the author.

In a document edited by Flanigan (1993), we 
have set forth an infrastructure strategy to achieve complex, integrated synthetic environments (such as synthetic battlespaces, synthetic manufacturing environments, and synthetic information-systems environments). The document provides a methodology to obtain integration, interoperability, portability, scalability, reusability, and realism for these environments. It also presents a technical administrative approach, data, applications, and technical (infrastructure) architectures, a synthetic-environments technical reference model (TRM) based upon the Defense TRM, technical and programmatic transition strategies, and a synchronization process for the development and modification of guiding documents.

Now resident within the DMSO IS is a protocols/standards module. It is designed to provide "browsing" overviews of protocols, standards, specifications, and areas of standardization (as well as where to get detailed help) for Department of Defense (DoD) program managers with imminent acquisitions. Overviews can be assessed on the basis of functional area (i.e., analysis, education, training and military operations, production and logistics, research and development, and test and evaluation), type of simulation (i.e., constructive-stand alone, constructive-distributed, live, virtual-stand alone, and virtual-distributed), and categories based upon those contained within the Defense TRM (i.e., simulation program interface, simulation platform services, and external environment interface). Included are approved and applicable DoD standards, Federal Information Processing Standards, voluntary international and national standards, other non-proprietary standards, industry-accepted product standards, and components and Ada-binding definitions from DoD and other reuse libraries and repositories. The module also presents a mini-tutorial on the types, hierarchy, limitations, use, and development of standards as well as a decision-aid chart for negotiating standards "thickets" and "wastelands".

\section{EMERGING PRODUCTS AND PROCESSES}

A dial-in, expert-system for developing standards profiles by DoD program managers with system-acquisition requirements may become operational early in the 1994 calendar year. When available, it will likely reside within the DMSO IS and be combined with or supersede the protocols/standards module described above.

There is also a growing need for an $M \& S$ standards framework. Such a framework would provide M\&S-community awareness of and conformance to existing standards. Further, it would identify areas where standards need to be formally adopted by DoD/Federal Government as well as provide migration paths (or road maps) to guide transitions from current conditions to standards-based operations. The framework is likely to be combined with the automated system for developing standards profiles.

A "fast track" DoD M\&S standards-adoption process is under consideration. The existing process for formal adoption of standards by DoD (under DoD Directive 4120.3-M, "Defense Standardization Program Policies and Procedures") can be time consuming and costly. Accordingly, this can raise the likelihood that informal adoption and use of nonstandard processes and mechanisms will occur by rapidly-evolving, $M \& S$-related efforts such as S\&TT Advanced Technology Demonstrations. A fast-track approach could be established by chartering a review committee for formally inserting into the DoD TRM appropriate M\&S standards/protocols proposed for DoD adoption. The resources of SIT may be utilized to establish the TRM review committee and to implement the committee's charter.

There is an rapidly emerging need for migrating toward seamless interoperability between DoD/FederalComponent M\&S IMs and electronic catalogs (so that "any call connects all"). An IM interoperability program may be initiated early in the 1994 calendar year and include fostering the standardization of IM data entry, data access, information contents, and screen-presentation formats.

Work has begun on an M\&S Data Administration/Standardization effort involving the Defense Information Systems Agency (DISA) and DMSO. It is directed at the problem of data being collected/generated/ manipulated by a diverse and growing community in a redundant and uncoordinated fashion that precludes data accessibility and reuse. This effort will be described in detail by C. Huo of DISA's Center for Standards in the DoD "Distributed Simulation" session.

\section{STANDARDS ROAD MAP}

A high-level, standards road map will be presented for discussion by the panel and to generate comments and suggestions from the audience. Copies will be made available prior to the start of the "DoD Infrastructure" session.

\section{REFERENCE}

Flanigan, W. F. 1993. Achieving integrated synthetic environments. In Synthetic Environments Strategic Plan Draft $3 B$, ed. J. Thorpe, 4.1-4.60. Alexandria, Virginia: Defense Modeling and Simulation Office. 


\section{AUTHOR BIOGRAPHY}

WILLIAM F. FLANIGAN, JR. is with the Defense Information Systems Agency's Center for Standards and on the Staff of the Defense Simulation and Modeling Office. He received a B.S. in electronics engineering from Clarkson College in 1961, an M.B.A. degree in corporation finance from Cornell University in 1963, an M.A degree in psychology from The George Washington University in 1968, and a Ph.D. degree in psychology from The University of Chicago in 1973. His interests are currently focused on the standardization of advanced distributed simulations and synthetic environments to enable their exploitation for National-defense preparedness and National-economy enhancement. 